-研究报告・

\title{
直刺变豆菜叶绿体全基因组及其特征
}

\author{
陈志祥 ${ }^{1}$ 姚雪莹 ${ }^{1}$ Stephen R. Downie ${ }^{2}$ 王奇志 ${ }^{*}$ \\ 1 (华侨大学化工学院园艺系, 福建厦门 361021) \\ 2 (Department of Plant Biology, University of Illinois at Urbana-Champaign, Urbana 61801)
}

\begin{abstract}
摘要: 直刺变豆菜(Sanicula orthacantha) 是中国广泛分布的多年生草本植物，也是一味著名的民族药。本文通过二 代高通量测序平台Illumina HiSeq PE150对直刺变豆菜叶绿体全基因组进行测序, 并通过生物信息学方法对其结构 特征进行分析。结果表明：直刺变豆菜叶绿体全基因组大小为 $157,163 \mathrm{bp}$, 包括大单拷贝区(large single copy, LSC)、小单拷贝区(small single copy, SSC)和 2 个反向重复序列(inverted repeat sequence, IRa和IRb), 长度分别为 87,547 bp、17,122 bp和26,247 bp, 具有典型被子植物叶绿体基因组环状四分体结构; 共注释得到129个基因, 包括8 个核糖体RNA (rRNA)基因、37个转运RNA (tRNA)基因和 84 个蛋白质编码基因。直刺变豆菜在叶绿体基因组结构、 基因种类、排列顺序上与其他伞形科植物基本一致。直刺变豆菜叶绿体全基因组测序的成功为变豆菜属植物完整 叶绿体基因组组装及其特征分析提供了新的方法。
\end{abstract}

关键词: 高通量测序; 变豆菜属; 基因组装; 系统发育分析

\section{Assembling and analysis of Sanicula orthacantha chloroplast genome}

\author{
Zhixiang Chen ${ }^{1}$, Xueying Yao ${ }^{1}$, Stephen R. Downie ${ }^{2}$, Qizhi Wang ${ }^{1 *}$ \\ 1 Department of Horticulture, Huaqiao University, Xiamen, Fujian 361021, China \\ 2 Department of Plant Biology, University of Illinois at Urbana-Champaign, Urbana 61801, USA
}

\begin{abstract}
Sanicula orthacantha is a perennial herb widely distributed in China and is a widely used for medicinal purposes. In this study, the whole chloroplast genome was sequenced and analyzed using bioinformatics methods. The results showed that it was a circular molecule 157,163 bp in length with a typical quadripartite structure including a pair of inverted repeats (IRa and IRb) of 26,247 bp that were separated by large and small single copy regions (LSC and SSC) of 87,547 bp and 17,122 bp, respectively. A total of 129 predicted genes, including 84 protein-coding genes, 37 tRNA genes and eight rRNA genes were identified. Compared with other Apiaeceae species, the $S$. orthacantha chloroplast genome had few differences in size, order and structure. The success of sequencing the $S$. orthacantha chloroplast genome provides a new method for the complete chloroplast genome assembly and characterization of Sanicula species, providing a methodological guide for the study of Sanicula plant evolution and phylogeny.
\end{abstract}

Key words: high-throughput sequencing; Sanicula L.; gene assembly; phylogenetic analysis

叶绿体普遍存在于陆地植物、藻类和部分原生 生物当中, 是绿色植物进行光合作用的细胞器, 具 有可自主遗传的基因组(邢少辰和Clarke, 2008)。裸 子植物中叶绿体基因组一般是父系遗传(Soltis et al, 2000), 而大多数被子植物的叶绿体基因组为母系 遗传, 其中大约 $20 \%$ 左右可能是双亲遗传或父系遗 传 (McCauley et al, 1996; SmallCronn \& Wendel,
2004)。近年来，随着测序技术的发展和测序成本的 降低, 已测序的叶绿体基因组数量迅速增加, 叶绿 体基因组的研究也得到了迅速发展, 对叶绿体基因 组的结构和变异有了更深入的了解。被子植物中叶 绿体基因组相对保守, 绝大多数为双链环状结构, 包括1个小的单拷贝区(small single copy, SSC)、1个 大的单拷贝区(large single copy, LSC)以及 1 对编码 
相同，方向相反的序列IRa和IRb (inverted repeats sequence, IR) (Zhang et al, 2012; 王玲等, 2012)。相比 核基因组, 叶绿体基因组具有结构相对保守、碱基 变异速率适中、易于测序等多种优势, 已广泛应用 于各植物类群的系统进化研究(Daniell et al, 2016)。

直刺变豆菜(Sanicula orthacantha, 异名黑鹅脚 板)是一味著名的民族药, 收录于《四川省中草药标 准(试行稿)》(四川省卫生局, 1979), 具有清热解毒、 益肺止咳、祛风除湿、活血通络等功效。但《全国 中草药汇编》、《中药大辞典》、《中国中药资源 志要》等药学书籍(张惠源, 1994; 谢宗万, 2014)记 载有同属数种植物混用的现象。随着高通量测序技 术的发展, 叶绿体基因组序列表现出较强分辨率 且适用于不同分类阶元的系统发育研究(Clegg et al, 1994; Downie \& Jansen, 2015), 可为药用植物物种鉴 定和混伪品鉴定提供更为准确的方法。但关于变豆 菜属物种叶绿体基因组数据未见报道, 限制了叶绿 体基因组在其物种鉴定和进化关系研究中的应用。

本研究以中国特有种直刺变豆菜为材料, 探索 适用于变豆菜属植物完整叶绿体基因组组装及特 征分析的方法, 为解决变豆菜同属植物系统进化和 易混淆种类提供参考。

\section{材料与方法}

\section{1 材料}

直刺变豆菜叶片于 2017 年4月采集于湖北省神 农架风景区 $\left(31^{\circ} 15^{\prime} \mathrm{N}, 109^{\circ} 56^{\prime} \mathrm{E}\right)$ 。凭证标本保存于 华侨大学园艺系标本室(00007622)。

\section{2 全基因组DNA提取及测序}

用植物基因组DNA试剂盒(上海生工)提取直刺 变豆菜全基因组DNA, 并送样测序。样品经百迈克 生物有限公司检测合格后, 采用 Illumina HiSeq PE150双末端测序策略进行建库, 建库类型为350 bp DNA小片段文库, 测序深度为10倍。

\section{3 叶绿体基因组组装及注释}

使用FastQC v0.11.7软件(Andrews, 2013)评估 测序原始的质量。在质量评估之后, 通过将所有原 始读数与目前已经报道的伞形科植物叶绿体全基 因组序列进行比对, 从中抽提直刺变豆菜叶绿体 reads, 同时基于比对结果选出覆盖度最好的序列,
即为最优参考序列。使用SOAPdenovo2 (Luo et al, 2012)将所有相关reads组装成重叠群, 再根据reads 的paired-end和overlap关系对组装结果进行优化。最 后使用NOVO-Plasty (Dierckxsens et al, 2017)对组装 结果进行补洞(Seed文件选用最优参考序列), 其平 均覆盖度达 $94.77 \%$ 。以最优参考序列为参考, 在软 件Geneious Basic 11.04 (Kearse et al, 2012)中使用原 核生物密码子对序列进行开放阅读框(open reading frame, ORF)预测并与参考序列进行比对注释，按照 预测的ORF中的终止密码子和起始密码子的位置 手动调整部分注释有误的蛋白编码基因。叶绿体基 因组 4 个边界注释是在Geneious Basic 11.04中使用 MAFFT将序列与其反向互补序列比对确认(首先确 定两个反向重复区)。此外, 使用Sequin软件完成直 刺变豆菜叶绿体基因组的提交。利用OGDRAW在线 工具(Lohse et al, 2013)绘制直刺变豆菜叶绿体基因 组环状图谱。

\section{4 叶绿体基因组数据分析}

利用MISA软件(Thiel et al, 2003)鉴定直刺变豆 菜叶绿体基因组中的简单重复序列(simple sequence repeats, SSR), 参数设置为 $1-10,2-6,3-5,4-5,5-5$, $6-5$, 即单核苷酸重复单元不少于 10 个, 二核苷酸重 复序列不少于 6 个，三核苷酸以上重复序列不少于 5 个。利用CodonW (Peden, 1999)计算同义密码子的 相对使用频率(relative synonymous codons usage, RSCU): RSCU > 1表示该密码子有偏好, RSCU $<1$ 表示该密码子使用率较低, RSCU $=1$ 表示该密码子 没有偏好性。

从GenBank下载前人已经发表的芹亚科和天胡 荽亚科(Ge et al, 2017)共15种物种的叶绿体基因组, 与本研究中直刺变豆菜叶绿体基因组序列进行比 对, 使用MAFFT (Kazutaka et al, 2002)比对, 比对结 果导入MEGA 7.0 (Kumar et al, 2016)软件, 采用最 大似然法(Maximum Likelihood, ML)构建系统发育 树。自举值(boostrap value)是基于1,000次抽样重复。

\section{5 系统发育分析}

伞形科分为芹亚科、变豆菜亚科、天胡荽亚科 (单人骅和余孟兰, 1999)。本研究从GenBank下载前 人已经发表的芹亚科和天胡荽亚科(Ge et al, 2017) 共15种物种的叶绿体基因组, 与本研究中直刺变豆 
菜叶绿体基因组序列进行比对。

\section{2 结果}

\section{1 叶绿体基因组组装与结构解析}

基于高通量测序技术得到全基因组，去掉低质 量reads后, 得到Clean data $10 \mathrm{G}$, 全基因组的GC含 量为 $36.58 \%$, 有效数据的Q20占 $97.56 \%$, 有效数据 的Q30占 $93.17 \%$ 。根据比对覆盖度发现棱子芹(Pleurospermum camtschaticum, NC_033343)为最优参
考序列，其覆盖度达 $94.77 \%$ ，组装后的叶绿体经检 验合格后，其叶绿体全基因组序列已在GenBank注 册，序列号为MK293943。

直刺变豆菜叶绿体基因组是共价闭合的双链 环状分子，包含LSC、SSC、IRa和IRb 4个区段(图1)。 序列全长 $157,163 \mathrm{bp}$, 其中LSC区段长 $87,547 \mathrm{bp}$, SSC区段长17,122 bp, 2 个IR区段均为26,247 bp, 且 GC含量为 $38.2 \%$ (图1)。

注释结果(表1)表明, 直刺变豆菜叶绿体基因

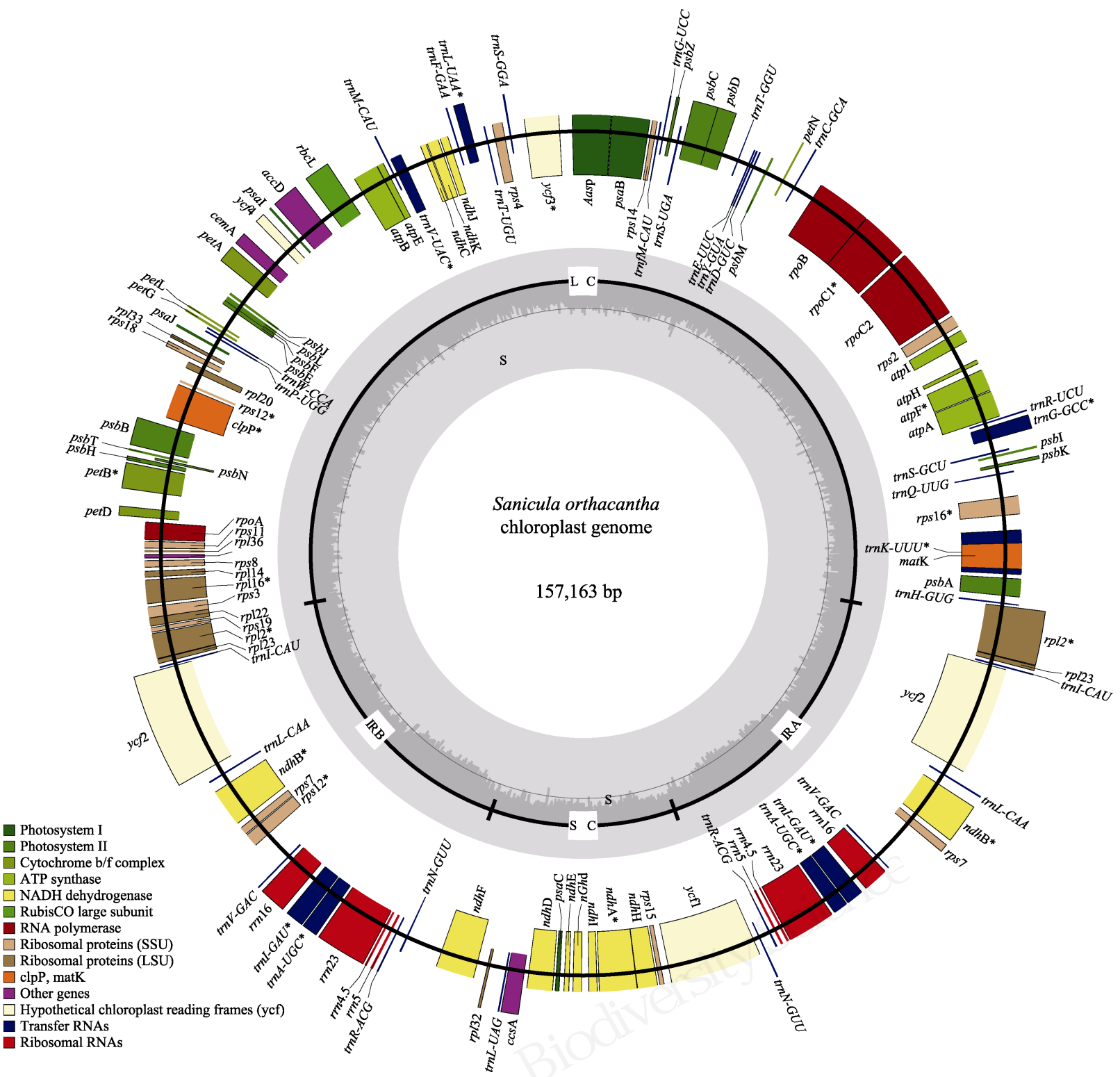

图1＼cjkstart直刺变豆菜叶绿体全基因组。外圈基因逆时针方向转录，内圈基因顺时针方向转录，不同的颜色表明基因不同的功能， 小圈中深灰色表示GC含量, 浅灰色表示AT含量。

Fig. 1 Sequence map of the Sanicula orthacantha chloroplast genome. Genes drawn outside of the circle are transcribed counter-clockwise, while genes shown on the inside of the circle are transcribed clockwise. Genes belonging to different functional groups are color-coded. The dark gray in the inner circle indicates GC content, while the light gray corresponds to AT content. 
表1 直刺变豆菜叶绿体基因组所编码的基因

Table 1 List of genes found in Sanicula orthacantha chloroplast genome

\begin{tabular}{|c|c|c|}
\hline $\begin{array}{l}\text { 基因分类 } \\
\text { Category for genes }\end{array}$ & $\begin{array}{l}\text { 基因分组 } \\
\text { Group of genes }\end{array}$ & $\begin{array}{l}\text { 基因名称 } \\
\text { Name of genes }\end{array}$ \\
\hline 表达相关基因 & 核糖体RNA基因 Ribosomal RNAs & $r r n 4.5(\times 2), r r n 5(\times 2), r r n 16(\times 2), r r n 23(\times 2)$ \\
\hline lication & 转运RNA基因 Transfer RNAs & 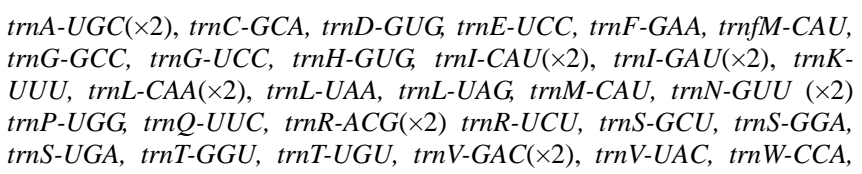 \\
\hline
\end{tabular}

核糖体小亚基基因

Ribosomal small subunit (SSU)

核糖体大亚基基因

Ribosomal large subuni (LSU)

RNA聚合酶亚基基因 RNA polymerase

\begin{tabular}{|c|c|c|}
\hline \multirow{7}{*}{$\begin{array}{l}\text { 光合作用相关基因 } \\
\text { Genes for } \\
\text { photosynthesis }\end{array}$} & 光合系统I基因 Photosystem I & psaA, psaB, psaC, psaI, psaJ \\
\hline & 光合系统II基因 Photosystem II & $\begin{array}{l}p s b \mathrm{~A}, p s b \mathrm{~B}, p s b \mathrm{~K}, p s b \mathrm{I}, p s b \mathrm{M}, p s b \mathrm{D}, p s b \mathrm{C}, p s b E, p s b \mathrm{~J}, p s b \mathrm{~L}, p s b \mathrm{~T}, p s b \mathrm{H}, \\
p s b \mathrm{~N}, p s b \mathrm{~F}, p s b \mathrm{Z}, p s b J\end{array}$ \\
\hline & 细胞色素复合物基因 Cytochrome b/f complex & pet $\mathrm{A}$, pet $\mathrm{D}$, pet $\mathrm{G}$, pet $\mathrm{L}$, pet $\mathrm{N}$ \\
\hline & ATP合酶基因 ATP synthase & $a t p \mathrm{~A}, a t p \mathrm{~F}, a t p \mathrm{H}, a t p \mathrm{I}, a t p \mathrm{E}, a t p \mathrm{~B}$ \\
\hline & $\begin{array}{l}\text { 依赖ATP的蛋白酶单元p基因 } \\
\text { ATP-dependent protease subunit p gene }\end{array}$ & $\operatorname{clpP}$ \\
\hline & $\begin{array}{l}\text { 二磷酸核酮糖羧化酶大亚基基因 } \\
\text { RubiscoCO large subunit }\end{array}$ & $r b c \mathrm{~L}$ \\
\hline & $\mathrm{NADH}$ 脱氢酶基因 NADH dehydrogenase & $n d h \mathrm{~J}, n d h \mathrm{~K}, n d h \mathrm{C}, n d h \mathrm{~B}(\times 2), n d h \mathrm{~F}, n d h \mathrm{D}, n d h \mathrm{E}, n d h \mathrm{G}, n d h \mathrm{I}, n d h \mathrm{~A}, n d h \mathrm{H}$ \\
\hline \multirow{5}{*}{$\begin{array}{l}\text { 其他基因 } \\
\text { Other genes }\end{array}$} & 成熟酶基因 Maturase & matK \\
\hline & 包裹膜蛋白基因 Envelop membrane protein & cemA \\
\hline & $\begin{array}{l}\text { 乙酰辅酶A羧化酶亚基基因 } \\
\text { Subunit of acetyl-CoA-carboxylase }\end{array}$ & $\operatorname{acc} \mathrm{D}$ \\
\hline & $\begin{array}{l}\text { c型细胞色素合成基因 } \\
\text { c-type cytochrome synthesis ccsA gene }\end{array}$ & $\operatorname{ccs} \mathrm{A}$ \\
\hline & $\begin{array}{l}\text { 转录起始因子基因 } \\
\text { Transcription initiation factor IF-1 }\end{array}$ & InfA \\
\hline $\begin{array}{l}\text { 未知功能基因 } \\
\text { Genes of unknown } \\
\text { function }\end{array}$ & $\begin{array}{l}\text { 保守开放阅读框 } \\
\text { Conserved open reading frames }\end{array}$ & $y c f 1, y c f 2(\times 2), y c f 3, y c f 4$ \\
\hline
\end{tabular}

rps16, rps2, rps14, rps4, rps18, rps11, rps8, rps3, rps19, rps7(×2), rps12, rps15

rpl33, rpl20, rpl36, rpl14, rpl16, rpl22, rpl2(×2), rpl23(×2), rpl32

rpoA, rpoB, rpoC1, rpoC2

psaA, psaB, psaC, psaI, psaJ

$p s b \mathrm{~A}, p s b \mathrm{~B}, p s b \mathrm{~K}, p s b \mathrm{I}, p s b \mathrm{M}, p s b \mathrm{D}, p s b \mathrm{C}, p s b E, p s b \mathrm{~J}, p s b \mathrm{~L}, p s b \mathrm{~T}, p s b \mathrm{H}$,

$a t p \mathrm{~A}, a t p \mathrm{~F}, a t p \mathrm{H}, a t p \mathrm{I}, a t p \mathrm{E}, a t p \mathrm{~B}$

$r b c \mathrm{~L}$
组共有129个基因, 包括 84 个蛋白编码基因、37个 tRNA基因和 8 个rRNA基因。其中，16个基因含有 2 个拷贝，包括5个蛋白编码的基因( $n d h \mathrm{~B} 、 r p l 2 、 r p s 7$ 、 $r p l 23 、 y c f 2) 、 7$ 个RNA基因(trnA-UGC、trnI-CAU、 trnI-GAU、trnL-CAA 、trnN-GUU、trnR-ACG、trnV$G A C)$ 和 4 个rRNA基因(rrn4.5、rrn5、rrn16、rrn23)。 蛋白编码基因序列总长77,439 bp, 占叶绿体基因组 序列的 $49.27 \%$ 。在蛋白编码基因中, 11 个基因含有 内含子(intron), 其中 7 个基因( $a t p F 、 n d h \mathrm{~A} 、 p e t \mathrm{~B}$ 、 $r p l 16 、 r p o C 1 、 r p s 12 、 r p s 16)$ 含有1个内含子, 另外 4 个基因( $c l p \mathrm{P} 、 n d h \mathrm{~B} 、 r p l 2 、 y c f 3)$ 含有 2 个内含子。

\section{2 叶绿体基因组SSRs开发}

本研究共鉴定到直刺变豆菜叶绿体基因组中
39 个SSR (附录1), 其中绝大部分SSR是单核苷酸重 复(28个，71.79\%)，双核苷酸重复有10个(25.64\%), 复杂重复有 1 个。除复杂重复SSR外, 其余SSRs均由 $\mathrm{A}$ 或 $\mathrm{T}$ 组成, 这表明 SSRs的碱基组成偏向使用 $\mathrm{A} / \mathrm{T}$ 碱基。

\section{3 叶绿体基因组密码子偏好性}

直刺变豆菜所有蛋白编码序列共含有 51,877 个 密码子(附录2)。其中，5,150个(9.93\%)编码亮氨酸， 708 个 $(1.36 \%)$ 编码甲硫氨酸, 它们分别是直刺变豆 菜叶绿体基因组中最多和最少的氨基酸, 并且 3 个 终止密码子中, UAA在直刺变豆菜叶绿体中使用最 为频繁(UGA 1.8\%; UAG 1.5\%; UAA 2.1\%), 直刺变 豆菜相对同义中密码子的使用(RSCU)见附录2。 


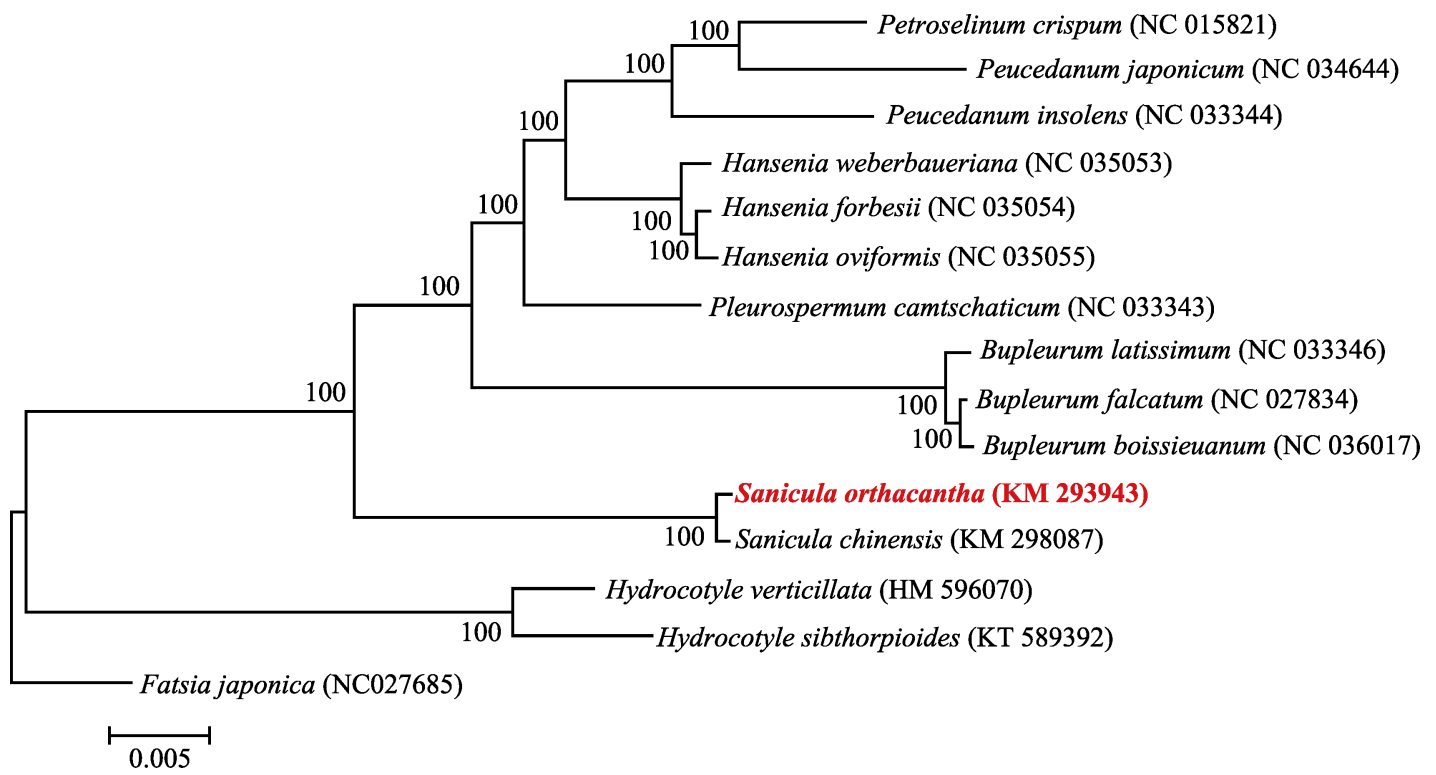

图2 基于叶绿体全基因组序列用最大似然法构建的15个物种的系统进化树

Fig. 2 Phylogenetic tree of 15 species based on chloroplast genome using Maximum Likelihood method

\section{4 系统发育分析}

系统发育树表明, 直刺变豆菜与变豆菜(S.chinensis)关系最近, 并与天胡荌亚科在聚同一分支(图2)。

\section{3 讨论}

本研究采用高通量测序技术对直刺变豆菜全 基因组DNA进行重测序, 根据比对结果, 选用棱子 芹叶绿体基因组为参考, 成功组装出完整的叶绿体 基因组。相比于传统方法, 从总DNA中提取叶绿体 基因组序列不需要单独提纯叶绿体DNA, 降低了实 验难度, 缩短了实验周期, 同时也未采用一代测序 进行补洞, 而是使用NOVO-Plasty软件进行补洞。 本研究建立了适用于该属植物完整叶绿体基因组 组装及其特征分析的方法, 为解决变豆菜同属植物 (软雀花(S.eleta)和川滇变豆菜(S.astrantiifolia)等)混 用提供了更准确的途径。

前人研究证明, 大部分被子植物叶绿体基因组 结构为高度保守的四分体结构(Jansen et al, 2005), 伞形科植物如人参(Panax ginseng) (Kim \& Lee, 2005)、胡萝卜(Ruhlman et al, 2006)等。本研究中直 刺变豆菜叶绿体基因组结构与上述伞形科植物叶 绿体基因组研究结果一致。

由于叶绿体基因组结构简单、相对保守且属于 母系遗传, 所以叶绿体简单重复序列是一种高效的 分子标记, 叶绿体基因组分子标记(cpSSRs)广泛应
用于属间分类、生物地理学和群体遗传学的研究 (Bayly et al, 2013; Zhao et al, 2015)。在直刺变豆菜 中, 我们发现了2种cpSSRs类型, 包括单碱基重复、 二碱基重复, 且这些SSRs中碱基重复单元均为 $\mathrm{A} / \mathrm{T}$; 同时也研究了直刺变豆菜叶绿体基因组密码子偏 好性, 这为变豆菜属植物叶绿体基因组研究提供了 更为详细、完善的资料, 为该属属间鉴定以及群体 和个体水平的遗传差异分析奠定基础。

本研究通过采用高通量测序的方法得到植物 全基因组DNA数据, 并使用NOVO-Plasty进行补洞, 成功组装出直刺变豆菜叶绿体基因组。此方法简 单、高效，但是应用时要注意以下几点: (1)在该物 种的科内外或属内外选择合适的参考基因组，以便 从全基因组序列中得到相关的叶绿体基因组序列; (2)所选的Seed文件为该物种 $r b c \mathrm{~L}$ 或最优参考序列, 或者根据gap位置选择亲缘关系较近物种同一位置 的序列。

\section{参考文献}

Andrews S (2013) Babraham Bioinformatics FastQC: A Quality Control Tool for High Throughput Sequence Data. http://www.bioinformatics.babraham.ac.uk/projects/fastqc.

Bayly MJ, Rigault P, Spokevicius A, Ladiges PY, Ades PK, Anderson C, Bossinger G, Merchant A, Udovicic F, Woodrow IE (2013) Chloroplast genome analysis of Australian eucalypts-Eucalyptus, Corymbia, Angophora, Allosyncarpia and Stockwellia (Myrtaceae). Molecular Phylogenetics 
\& Evolution, 69, 704-716.

Clegg MT, Gaut BS, Learn G, Morton BR (1994) Rates and patterns of chloroplast DNA evolution. Proceedings of the National Academy of Sciences, USA, 91, 6795-6801.

Daniell H, Lin CS, Ming Y, Chang WJ (2016) Chloroplast genomes: Diversity, evolution, and applications in genetic engineering. Genome Biology, 17, 134-163.

Dierckxsens N, Mardulyn P, Smits G (2017) NOVO-Plasty: De novo assembly of organelle genomes from whole genome data. Nucleic Acids Research, 45, e18.

Downie SR, Jansen RK (2015) A comparative analysis of whole plastid genomes from the Apiales: Expansion and contraction of the inverted repeat, mitochondrial to plastid transfer of DNA, and identification of highly divergent noncoding regions. Systematic Botany, 40, 336-351.

Ge L, Shen LQ, Chen QY, Li XM, Zhang L (2017) The complete chloroplast genome sequence of Hydrocotyle sibthorpioides (Apiales: Araliaceae). Mitochondrial DNA Part B, 2, 29-30.

Jansen RK, Raubeson LA, Boore JL, Depamphilis CW, Chumley TW, Haberle RC, Wyman SK, Alverson AJ, Peery R, Herman SJ (2005) Methods for obtaining and analyzing whole chloroplast genome sequences. Methods in Enzymology, 395, 348-384.

Kazutaka K, Kazuharu M, Kei-Ichi K, Takashi M (2002) MAFFT: A novel method for rapid multiple sequence alignment based on fast Fourier transform. Nucleic Acids Research, 30, 3059-3066.

Kearse M, Moir R, Wilson A, Stoneshavas S, Cheung M, Sturrock S, Buxton S, Cooper A, Markowitz S, Duran C (2012) Geneious Basic: An integrated and extendable desktop software platform for the organization and analysis of sequence data. Bioinformatics, 28, 1647-1649.

Kim KJ, Lee HL (2005) Complete chloroplast genome sequences from Korean ginseng (Panax schinseng Nees) and comparative analysis of sequence evolution among 17 vascular plants. DNA Research, 11, 247-261.

Kumar S, Stecher G, Tamura K (2016) MEGA7: Molecular Evolutionary Genetics Analysis version 7.0 for bigger datasets. Molecular Biology \& Evolution, 33, 1870-1874.

Lohse M, Drechsel O, Kahlau S, Bock R (2013) OrganellarGenomeDRAW - A suite of tools for generating physical maps of plastid and mitochondrial genomes and visualizing expression data sets. Nucleic Acids Research, 41, W575.

Luo R, Liu B, Xie Y, Li Z, Huang W, Yuan J, He G, Chen Y, Qi P, Liu Y (2012) SOAPdenovo2: An empirically improved memory-efficient short-read de novo assembler. GigaScience, $1,18$.

McCauley DE, Stevens JE, Peroni PA, Raveill JA (1996) The spatial distribution of chloroplast DNA and allozyme polymorphisms within a population of Silene alba (Caryophyllaceae). American Journal of Botany, 83, 727-731.
Peden JF (1999) CodonW. PhD Dissertation, University of Nottingham, Nottinghamshire, UK.

Ruhlman T, Lee SB, Jansen RK, Hostetler JB, Tallon LJ, Town CD, Daniell H (2006) Complete plastid genome sequence of Daucus carota: Implications for biotechnology and phylogeny of angiosperms. BMC Genomics, 7, 222-235.

Shan RH, She ML (1979) Flora Reipublicae Popularis Sinicae, Tomus 14, pp. 12-67. Science Press, Beijing. (in Chinese) [单人骅, 余孟兰 (1979) 中国植物志, 第十四卷, 12-67 页. 科学出版社, 北京.]

Sichuan Provincial Health Department (1979) Sichuan Chinese Herbal Medicine Standard (Trial Draft). Sichuan Provincial Health Department, Chengdu. (in Chinese) [四川省卫生局 (1979) 四川省中草药标准(试行稿). 四川省卫生局, 成都.]

Small RL, Cronn RC, Wendel JF (2004) Use of nuclear genes for phylogeny reconstruction in plants. Australian Systematic Botany, 17, 145-170.

Soltis PS, Soltis DE (2000) The role of genetic and genomic attributes in the success of polyploids. Proceedings of the National Academy of Sciences, USA, 97, 7051-7057.

Thiel T, Michalek W, Varshney R, Graner A (2003) Exploiting EST databases for the development and characterization of gene-derived SSR-markers in barley (Hordeum vulgare L.). Theoretical \& Applied Genetics, 106, 411-422.

Wang L, Dong WP, Zhou SL (2012) Structural mutations and reorganizations in chloroplast genomes of flowering plants. Acta Botanica Boreali-Occidentalia Sinica. 32, 1282-1288. (in Chinese with English abstract) [王玲, 董文攀, 周世良 (2012) 被子植物叶绿体基因组的结构变异研究进展. 西 北植物学报, 32, 1282-1288.]

Xie ZW (1996) The Compilation of Chinese Herbal Medicine. People’s Medical Publishing House, Beijing. (in Chinese) [谢宗万 (1996) 全国中草药汇编. 人民卫生出版社, 北京.]

Xing SC, Clarke JL (2008) Process in chloroplast genome analysis. Progress in Biochemistry and Biophysics, 35, 21-28. (in Chinese with English abstract) [邢少辰, Clarke JL (2008) 叶绿体基因组研究进展. 生物化学与生物物理 进展, 35, 21-28.]

Zhang HY (1994) Annals of Chinese Traditional Medicine Resources. Science Press, Beijing. (in Chinese) [张惠源 (1994) 中国中药资源志要. 科学出版社, 北京.]

Zhang T, Fang Y, Wang X, Deng X, Zhang X, Hu S, Yu J (2012) The complete chloroplast and mitochondrial genome sequences of Boea hygrometrica: Insights into the evolution of plant organellar genomes. PLoS ONE, 7, e30531.

Zhao YB, Yin JL, Guo HY, Zhang YY, Xiao W, Sun C, Wu JY, Qu XB, Yu J, Wang XM, Xiao JF (2015) The complete chloroplast genome provides insight into the evolution and polymorphism of Panax ginseng. Frontiers in Plant Science, 5, 696-709.

(责任编委：郭亚龙 责任编辑：时意专) 


\section{附录 Supplementary Material}

\section{附录1 直刺变豆菜叶绿体基因组中的SSR}

Appendix 1 SSR in the chloroplast genome of Sanicula orthacantha

http://www.biodiversity-science.net/fileup/PDF/2018332-1.pdf

附录2 直刺变豆菜叶绿体基因组密码子使用及反密码子的鉴定

Appendix 2 Codon usage and codon-anticodon recognition pattern for tRNA in Sanicula orthacantha

http://www.biodiversity-science.net/fileup/PDF/2018332-2.pdf 
陈志祥, 姚雪莹, Stephen R. Downie, 王奇志. 直刺变豆菜叶绿体全基因组及其特征分析, 生物多样性, 2019, 27(4): 366-372.

附录1 直刺变豆菜叶绿体基因组中的SSR

Appendix 1 SSR in the chloroplast genome of Sanicula orthacantha

\begin{tabular}{|c|c|c|c|c|c|c|c|c|c|c|c|}
\hline $\begin{array}{l}\text { 编号 } \\
\text { No. }\end{array}$ & $\begin{array}{l}\text { 类型 } \\
\text { type }\end{array}$ & $\begin{array}{l}\text { 简单重复序列 } \\
\text { SSR }\end{array}$ & $\begin{array}{l}\text { 大小 } \\
\text { size }\end{array}$ & $\begin{array}{l}\text { 起始 } \\
\text { start }\end{array}$ & $\begin{array}{l}\text { 终止 } \\
\text { end }\end{array}$ & $\begin{array}{l}\text { 编号 } \\
\text { No. }\end{array}$ & $\begin{array}{l}\text { 类型 } \\
\text { type }\end{array}$ & $\begin{array}{l}\text { 简单重 } \\
\text { SSR }\end{array}$ & $\begin{array}{l}\text { 大小 } \\
\text { size }\end{array}$ & $\begin{array}{l}\text { 起始 } \\
\text { start }\end{array}$ & $\begin{array}{l}\text { 终止 } \\
\text { end }\end{array}$ \\
\hline 1 & p2 & (TA)8 & 16 & 146 & 161 & 21 & $\mathrm{p} 1$ & (T)10 & 10 & 67079 & 67088 \\
\hline 2 & $\mathrm{p} 1$ & (A)10 & 10 & 3918 & 3927 & 22 & p2 & (TA)7 & 14 & 68364 & 68377 \\
\hline 3 & p2 & $(\mathrm{AT}) 6$ & 12 & 10598 & 10609 & 23 & $\mathrm{p} 1$ & (T)11 & 11 & 72233 & 72243 \\
\hline 4 & $\mathrm{p} 1$ & $(\mathrm{~T}) 10$ & 10 & 13205 & 13214 & 24 & $\mathrm{p} 1$ & (A)10 & 10 & 72394 & 72403 \\
\hline 5 & $\mathrm{p} 1$ & (A)11 & 11 & 13832 & 13842 & 25 & $\mathrm{p} 1$ & (A)11 & 11 & 73034 & 73044 \\
\hline 6 & $\mathrm{p} 1$ & (T)13 & 13 & 16497 & 16509 & 26 & $\mathrm{p} 1$ & (T)10 & 10 & 80002 & 80011 \\
\hline 7 & $\mathrm{p} 1$ & (A)11 & 11 & 17338 & 17348 & 27 & $\mathrm{p} 1$ & (T)12 & 12 & 81934 & 81945 \\
\hline 8 & $\mathrm{p} 1$ & (T)11 & 11 & 19571 & 19581 & 28 & p2 & (AT)9 & 18 & 85809 & 85826 \\
\hline 9 & $\mathrm{p} 1$ & (A)10 & 10 & 23660 & 23669 & 29 & $\mathrm{p} 1$ & (T)14 & 14 & 86134 & 86147 \\
\hline 10 & $\mathrm{p} 1$ & $(\mathrm{~T}) 12$ & 12 & 27306 & 27317 & 30 & $\mathrm{p} 1$ & (T)15 & 15 & 100230 & 100244 \\
\hline 11 & p2 & (TA)8 & 16 & 28606 & 28621 & 31 & $\mathrm{p} 1$ & (T)10 & 10 & 104118 & 104127 \\
\hline 12 & p2 & (AT)8 & 16 & 30690 & 30705 & 32 & $\mathrm{p} 1$ & (T)12 & 12 & 115566 & 115577 \\
\hline 13 & $\mathrm{p} 1$ & (A)11 & 11 & 32439 & 32449 & 33 & p2 & (TA)6 & 12 & 120806 & 120817 \\
\hline 14 & c & $\begin{array}{l}\text { (T)10aaattctaacag } \\
\text { (AT)6 }\end{array}$ & 34 & 33642 & 33675 & 34 & p2 & (AT)8 & 16 & 121041 & 121056 \\
\hline 15 & p2 & $(\mathrm{TA}) 7$ & 14 & 48107 & 48120 & 35 & $\mathrm{p} 1$ & (T)15 & 15 & 127797 & 127811 \\
\hline 16 & $\mathrm{p} 1$ & (T)10 & 10 & 52915 & 52924 & 36 & $\mathrm{p} 1$ & (T)11 & 11 & 128081 & 128091 \\
\hline 17 & $\mathrm{p} 1$ & (T)10 & 10 & 55963 & 55972 & 37 & $\mathrm{p} 1$ & (A)10 & 10 & 137617 & 137626 \\
\hline 18 & $\mathrm{p} 1$ & (T)10 & 10 & 60795 & 60804 & 38 & $\mathrm{p} 1$ & (A)15 & 15 & 141500 & 141514 \\
\hline 19 & p2 & (TA)6 & 12 & 63466 & 63477 & 39 & $\mathrm{p} 1$ & (A)14 & 14 & 155597 & 155610 \\
\hline 20 & $\mathrm{p} 1$ & (A)10 & 10 & 65030 & 65039 & & & & & & \\
\hline
\end{tabular}

p1、p2分别代表该SSR的类型为mononucleotide、dinucleotide; c代表该SSR类型是complex. 
陈志祥, 姚雪芗, Stephen R. Downie, 王奇志. 直刺变豆菜叶绿体全基因组及其特征分析, 生物多样性, 2019, 27(4): 366-372.

附录2 直刺变豆菜叶绿体基因组密码子使用及反密码子的鉴定

Appendix 2 Codon usage and codon-anticodon recognition pattern for tRNA in the Sanicula orthacantha

\begin{tabular}{|c|c|c|c|c|c|c|c|c|c|c|c|c|c|c|c|}
\hline 氨基酸 & 密码子 & 大小 & 密码 & 子氨基酸 & 密码子 & 大小 & 密码 子 & 氨基酸 & 密码子 & 大小 & 密码于 & 子氨基酸 & 密码子 & 大小 & 密码子 \\
\hline $\begin{array}{l}\text { Amino } \\
\text { acid }\end{array}$ & Codon & Number & $\begin{array}{l}\text { 使 用 } \\
\text { 对概率 }\end{array}$ & $\begin{array}{l}\text { 相Amino } \\
\text { acid }\end{array}$ & Codon & Number & $\begin{array}{l}\text { 使 用 相 } \\
\text { 对概率 }\end{array}$ & $\begin{array}{l}\text { Amino } \\
\text { acid }\end{array}$ & Codon & Number & $\begin{array}{l}\text { 使 用 } \text { 极 } \\
\text { 对概率 }\end{array}$ & $\begin{array}{l}\text { 目Amino } \\
\text { acid }\end{array}$ & Codon & Number & $\begin{array}{l}\text { 使用 相 } \\
\text { 对概率 }\end{array}$ \\
\hline$\overline{\text { Phe }}$ & UUU & 2162 & 1.19 & Ser & $\overline{\mathrm{UCU}}$ & 1168 & 1.47 & \begin{tabular}{|l|} 
Tyr \\
\end{tabular} & UAU & 1408 & 1.35 & Cys & UGU & 675 & 1.23 \\
\hline & UUC & 1458 & 0.81 & & UCC & 914 & 1.15 & & UAC & 674 & 0.65 & & UGC & 422 & 0.77 \\
\hline Leu & UUA & 1045 & 1.22 & & UCA & 894 & 1.12 & TER & UAA & 1086 & 1.16 & TER & UGA & 934 & 1.00 \\
\hline & UUG & 1104 & 1.29 & & UCG & 618 & 0.78 & & UAG & 778 & 0.83 & Trp & UGG & 708 & 1.00 \\
\hline & CUU & 1100 & 1.28 & Pro & $\mathrm{CCU}$ & 698 & 1.09 & His & CAU & 908 & 1.41 & Arg & CGU & 416 & 0.74 \\
\hline & CUC & 655 & 0.76 & & CCC & 641 & 1.00 & & CAC & 381 & 0.59 & & CGC & 255 & 0.45 \\
\hline & CUA & 734 & 0.86 & & CCA & 777 & 1.21 & Gln & CAA & 1025 & 1.39 & & CGA & 569 & 1.01 \\
\hline & CUG & 512 & 0.60 & & CCG & 451 & 0.70 & & CAG & 455 & 0.61 & & CGG & 384 & 0.68 \\
\hline Ile & AUU & 1737 & 1.22 & Thr & $\mathrm{ACU}$ & 707 & 1.19 & Asn & AAU & 1706 & 1.36 & Ser & AGU & 669 & 0.84 \\
\hline & AUC & 1123 & 0.79 & & ACC & 581 & 0.98 & & AAC & 794 & 0.64 & & AGC & 520 & 0.65 \\
\hline & AUA & 1424 & 1.00 & & ACA & 701 & 1.18 & Lys & AAA & 2088 & 1.33 & Arg & AGA & 1098 & 1.95 \\
\hline Met & AUG & 818 & 1.00 & & ACG & 383 & 0.65 & & AAG & 1044 & 0.67 & & AGG & 655 & 1.18 \\
\hline Val & GUU & 797 & 1.38 & Ala & GCU & 541 & 1.29 & Asp & GAU & 1102 & 1.43 & Gly & GGU & 585 & 1.01 \\
\hline & GUC & 419 & 0.73 & & GCC & 390 & 0.93 & & GAC & 438 & 0.57 & & GGC & 367 & 0.64 \\
\hline & GUA & 683 & 1.18 & & GCA & 467 & 1.12 & Glu & GAA & 1375 & 1.38 & & GGA & 798 & 1.38 \\
\hline & GUG & 409 & 0.71 & & GCG & 276 & 0.66 & & GAG & 616 & 0.62 & & GGG & 557 & 0.98 \\
\hline
\end{tabular}

RSCU: 密码子使用相对概率 Relative synonymous codons usage 